\title{
Thermodynamics of the interaction between SARS-CoV-2 spike protein and human ACE2 receptor. Effects of possible ligands.
}

\author{
Cristina García-Iriepa ${ }^{1,2}$, Cécilia Hognon³, Antonio Francés-Monerris ${ }^{3,4}$, Isabel Iriepa ${ }^{2,5}$, Tom \\ Miclot ${ }^{3,6}$, Giampaolo Barone ${ }^{6}$, Antonio Monari ${ }^{3 *}$, Marco Marazzi ${ }^{1,2 *}$
}

\author{
${ }^{1}$ Department of Analytical Chemistry, Physical Chemistry and Chemical Engineering, Universidad de Alcalá, Ctra. Ma- \\ drid-Barcelona, Km 33,600, 28871 Alcalá de Henares, Madrid, Spain. \\ ${ }^{2}$ Chemical Research Institute "Andrés M. del Río" (IQAR), Universidad de Alcalá, 28871 Alcalá de Henares, Madrid, \\ Spain. \\ 3 Université de Lorraine and CNRS, LPCT UMR 7019, F-54000 Nancy, France. \\ ${ }^{4}$ Departament de Química Física, Universitat de València, 46100 Burjassot, Spain. \\ ${ }^{5}$ Department of Organic and Inorganic Chemistry, Universidad de Alcalá, Ctra. Madrid-Barcelona, Km 33,600, 28871 \\ Alcalá de Henares, Madrid, Spain. \\ ${ }^{6}$ Department of Biological, Chemical and Pharmaceutical Sciences and Technologies, Università degli Studi di Palermo, \\ Viale delle Scienze, 90128 Palermo, Italy.
}

\begin{abstract}
Since the end of 2019, the coronavirus SARS-CoV-2 has caused more than 180,000 deaths all over the world, still lacking a medical treatment despite the concerns of the whole scientific community. Human Angiotensin-Converting Enzyme 2 (ACE2) was recently recognized as the transmembrane protein serving as SARS-CoV-2 entry point into cells, thus constituting the first biomolecular event leading to COVID-19 disease. Here, by means of a state-of-the-art computational approach, we propose a rational evaluation of the molecular mechanisms behind the formation of the complex and of the effects of possible ligands. Moreover, binding free energy between ACE2 and the active Receptor Binding Domain (RBD) of the SARS-CoV-2 spike protein is evaluated quantitatively, assessing the molecular mechanisms at the basis of the recognition and the ligand-induced decreased affinity. These results boost the knowledge on the molecular grounds of the SARS-CoV-2 infection and allow to suggest rationales useful for the subsequent rational molecular design to treat severe COVID-19 cases.
\end{abstract}

A novel strain of coronavirus inducing severe acute respiratory disease (SARS) developed at the end of 2019 in mainland China and was later identified as SARS-CoV-2. Since then, after readily diffusing in Eastern Countries, SARS-CoV-2 has been at the origin of the outbreak of a severe pandemic, Covid-19, at present widespread in all the continents. ${ }^{1-4}$ Strict social distancing and lock down measures have since been implemented to contain the diffusion of Covid-19 and the pressure it exerts on public health systems, due to the possible development of acute respiratory stress and bilateral pneumonia, requiring appropriate intensive care treatment. ${ }^{5-7}$ Indeed, although the mortality ratio of Covid-19 is relatively low, compared to other related diseases, and usually associated with other preexistent morbidity, the very high transmissibility ratio, also due to a large number of asymptomatic patients, is related to a very fast-growing rate of the infection. ${ }^{8-10}$ At the moment of the preparation of this manuscript, Covid-19 has infected more than 2 million persons worldwide, causing more than 180,000 deaths, and after having severely affected Asia and Europe, is rapidly growing in the United States and in the American continent. ${ }^{11}$ However, at present no real definitive therapeutic strategy is available to counteract the infection from SARS-CoV-2.

Due to the unprecedented severity of the sanitary crisis, and of its strong impact on both social and economic life, important scientific efforts have been devoted to model and comprehend the action of the virus and the outcome of the infection. In particular, the genome of the virus has been rapidly sequenced,12,13 and in parallel, the structure of its main protein apparatus has been resolved,14-16 especially using cryogenic Electron 
Microscopy (cryoEM) techniques. ${ }^{17}$ Molecular modeling and simulation studies have also been performed to rationalize, at atomistic level, the behavior of the different involved proteins, ${ }^{18}$ the interactions pattern between them and other biological structures such as nucleic acids, ${ }^{19}$ and finally the inherent differences between SARS-CoV-2 proteome and the ones of other coronaviruses, such as SARS-CoV or the Middle East Respiratory Syndrome (MERS) agents. ${ }^{20}$

Among the varied proteic apparatus of SARS-CoV-2, special attention has been devoted to the spike protein. This large protein includes a transmembrane domain protruding from the surface of the viral envelop, used by the virus to recognize the host cell. ${ }^{21}$ Indeed, after binding to the human receptors, via its specific Receptor Binding Domain (RBD), the large conformation changes induced allow the fusion of the viral and the host membranes, which represents the first step of the infection, i.e. the entry of the viral material into healthy cells. High resolution structures of the full spike protein complex have been obtained, also resolving different conformational states of RBD, namely the active open conformations, the semi-active and the closed state. ${ }^{17}$

The molecular target of the spike protein of coronaviruses in general and SARS-CoV-2 in particular, their entry gate, has been recognized in the Angiotensin Converter Enzyme 2 (ACE2, Figure 1). ${ }^{22}$ ACE2 is largely present in the external membranes of cells belonging to different human organs, such as lungs, kidneys, and intestine and has a fundamental role in regulating blood pressure level. ${ }^{23,24}$ In addition, it has also a secondary role in regulating membrane trafficking of neutral amino acid transporters. ${ }^{25}$ The interaction with ACE2, and consequently the inhibition of its biological functions, has also been recognized as one of the reasons of the high morbidity of SARS viruses. ${ }^{26-28}$ As a matter of fact, ACE2 is regarded as a favorable target of potential therapeutic agents counteracting SARS-CoV-2 infectivity limiting its harmful effects. Consequently, high resolution structures of the complex between RBD and the extramembrane domain of ACE2 (RBD/ACE2) have been obtained.22 The main interaction patterns driving the formation of the RBD/ACE2 complex have also been pointed out and rationalized, highlighting the crucial differences with other coronaviruses. The hotspots assuring the efficient recognition by RBD have been identified in the so-called peptide domain (PD) of the ACE2 receptor (Figure 1), consisting of an extended $\alpha$-helix region, and traced back to the formation of a dense hydrogen bonding network with RBD. ${ }^{17}$

An efficient therapeutic strategy hence could rely on the inhibition, by putative drugs, of the ACE2 PD domain to prevent the formation, or at least strongly destabilize, the RBD/ACE2 complex to reduce the virus infecting potential as schematized in Figure 1. ACE2 is known to act as a glycoprotein developing favorable interactions with sugar moieties, ${ }^{29}$ that could also favorably compete with RBD in establishing hydrogen bonds with the PD site. For this reason, in this contribution, we analyze, by using a fully multiscale approach, the possible interaction of glycosylated potential therapeutic agents with ACE2 and their inhibition capacity over the PD domain.

In particular, as illustrated in Figure $1 \mathrm{~b}$, we considered two classes of compounds widely available and already used in clinical applications: antibiotics based on aureolic acids (plicamycin, chromomycin A3, and UCH9) and flavonoids (diosmin, rutin, and naringin). First, we have performed molecular docking studies to assess the presence of suitable binding poses leading to possible PD inhibition. Second, the effects of the drug binding on the RBD/ACE2 complex stability and dynamic behavior have been revealed using equilibrium allatom molecular dynamics (MD) simulations. Finally, the thermodynamic binding free energy of the RBD/ACE2 complex has been obtained for the first time in presence and absence of the potentially inhibiting drugs, providing a quantification of the destabilizing effects of our lead compound. 


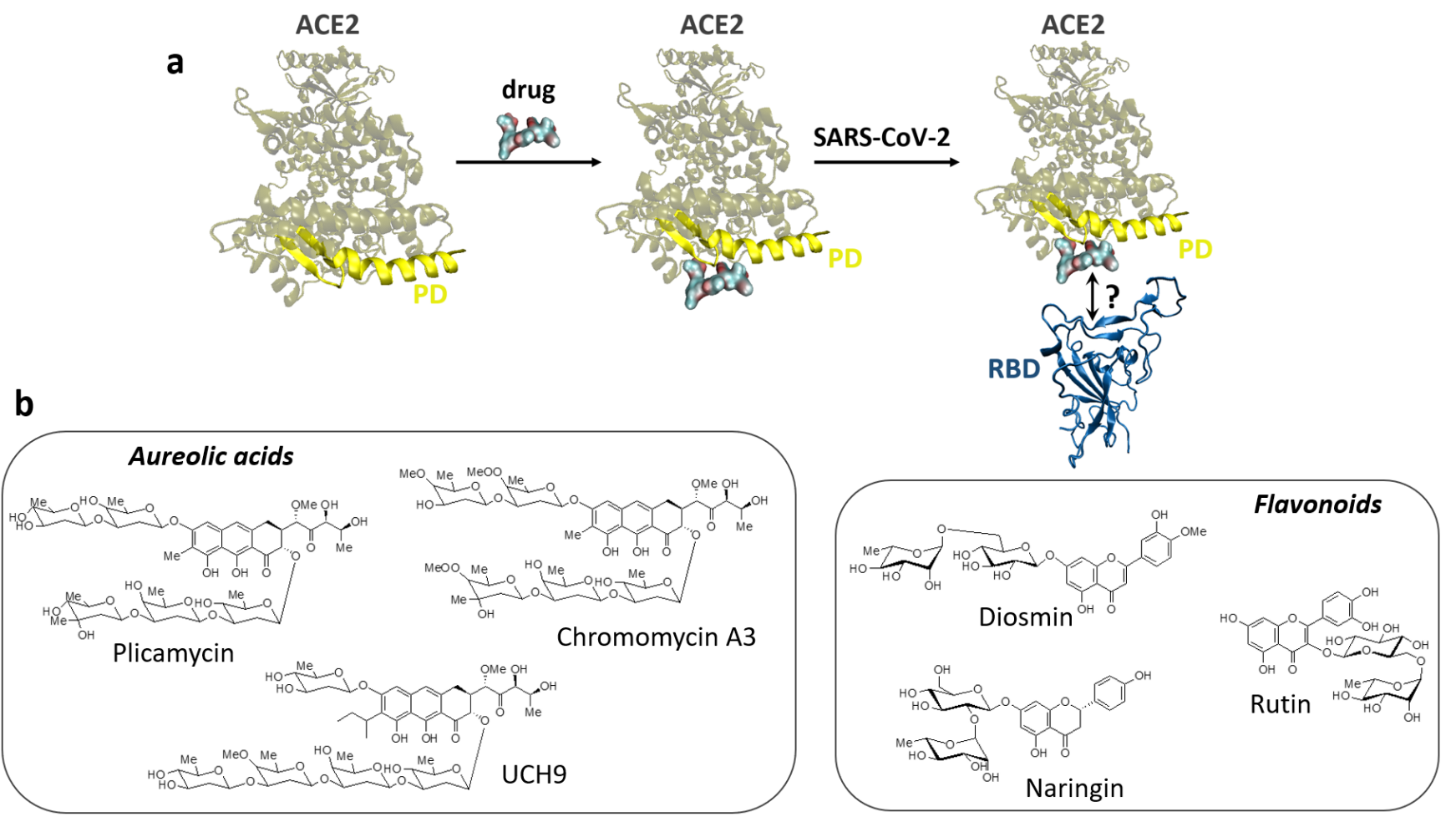

Fig. 1 | Can we lock the SARS-CoV-2 cell gate in presence of drugs? a, Depiction of human Angiotensin-Converting Enzyme 2 (ACE2) considering possible interactions of its Peptide Domain (PD) with administered drugs, that could in turn limit or avoid SARSCoV-2 recognition through its active Receptor Binding Domain (RBD). b, Structures of the drugs under study: aureolic acids, including plicamycin, chromomycin A3, and UCH9; flavonoids, including diosmin, rutin, and naringin.

\section{Results}

The results of the flexible drug docking are reported in Figure 2 and more extensively in Supplementary Figure 1. All the chosen compounds are previewed to form stable aggregates with ACE2, although slight differences in the binding energies are evidenced. Importantly, four main interaction hot spots are identified encompassing different regions of the enzyme (Figure 2). The results of the docking indicate that the four regions are generally competitive for all the compounds under study. Three of them are significant in terms of RBD/ACE2 inhibition purposes, whereas only one site is clearly out of reach of the RBD interaction area and is instead situated close to the ACE2 catalytic region ${ }^{21}$ (Figure 2 dark grey). For obvious structural reasons, this interacting site is most unlikely to significantly perturb the binding with RBD and hence is not considered in the following. 


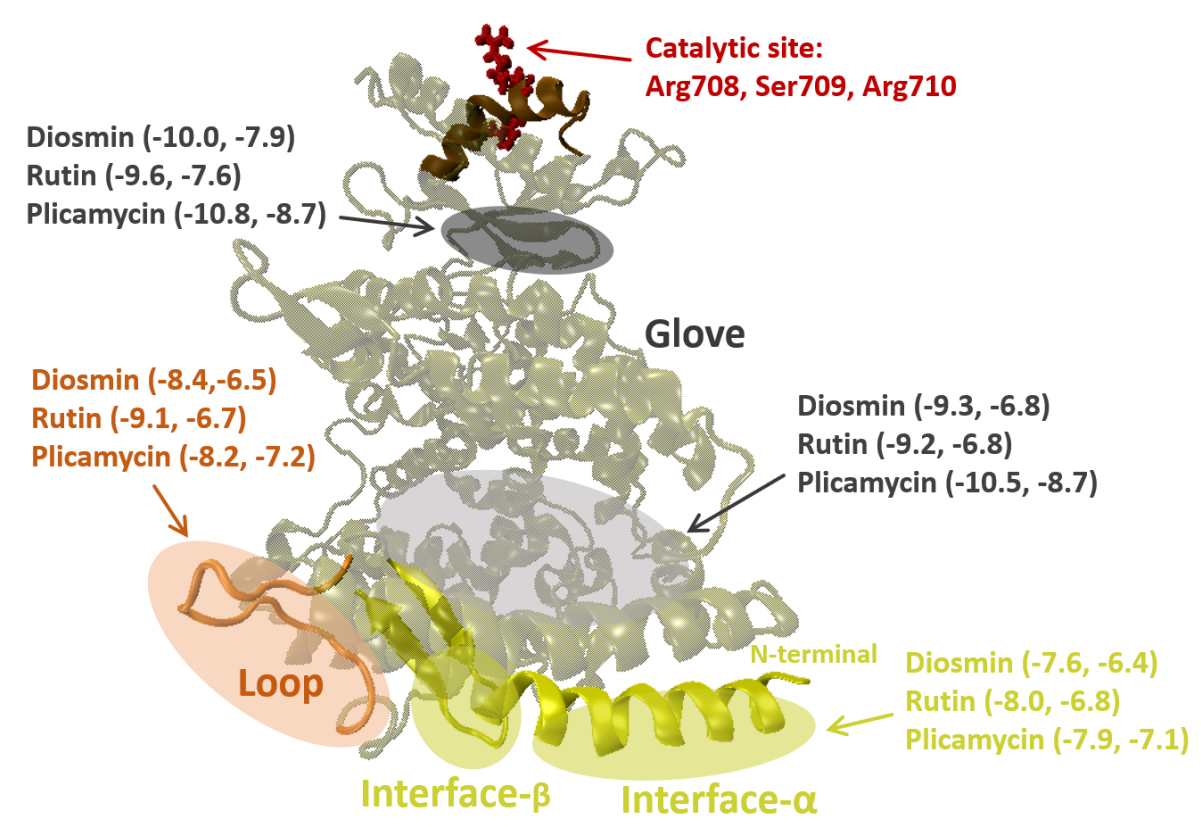

Fig. 2 | Glycosilated drugs preserve the ACE2 catalytic function. Different domains of the ACE2: the Peptide Domain, PD, (yellow) formed by an N-terminal $\alpha$-helix and a 2 -strands $\beta$-sheet, forming the potential interface region with the RBD; the loop at the side of the interface region (orange); the glove domain bridging interface region and the catalytic site (dark red), near the C-terminus. For each drug, the binding sites together with the range of binding energy affinities are shown in kcal/mol, as resulted from the docking study.

On the other hand, the residual three residual interacting sites, lie close to the RBD binding region. The glove site (light grey in Figure 2) constitutes a slightly buried pocket formed by ACE2 $\alpha$-helices positioned just on top of the PD domain. The loop domain (orange in Figure 2) is mainly constituted by an unstructured loop lying close to the RBD upon the formation of the complex. Finally, two sites are identified directly positioned on the N-terminal PD area (yellow in Figure 2) and named respectively interface- $\alpha$ and interface- $\beta$. Interestingly, while interface- $\alpha$ can be observed for all the docked compounds, interface- $\beta$ is mainly occupied by aureolic acids and plicamycin in particular. Obviously, these latter sites clearly represent the most promising candidates for ACE2 inhibition since they are susceptible to strongly perturb the recognition and binding of RBD. Finally, it is important to point out that no specific interaction with the ACE2 catalytic active site, composed of the amino acid triad Arg708, Ser709, and Arg710, ${ }^{21}$ has been observed. This fact is extremely important since, while blocking the RBD/ACE2 formation is supposed as most beneficial, the inhibition of the native catalytic activity of the enzyme should be avoided to limit severe side effects of the drug.

On the basis of the docking results, and in order to provide a reasonable sampling and description of the effects produced by the different modes, we have chosen three compounds to perform equilibrium MD simulations of the ACE2/RBD complex in presence of the drug, namely diosmin, rutin and plicamycin. For each of these compounds, three independent MD trajectories have been obtained, starting from initial conditions corresponding to different binding poses: glove and loop sites, interface- $\alpha$ and interface- $\beta$. MD of the native RBD/ACE2 complex in the absence of any ligand was also performed for comparison. In all cases, the equilibrium MD yielded stable and persistent aggregates between the RBD/ACE2 complex and the drugs, as evidenced by the value of the Root Mean Square Deviation (RMSD) reported in Supplementary Figure 6, and by the fact that neither the macroscopic disruption of the RBD/ACE2 complex nor the ejection of the drug was observed. However, important differences can be observed depending on the individual drugs and on the specific interaction site, as illustrated in Figure 3. 

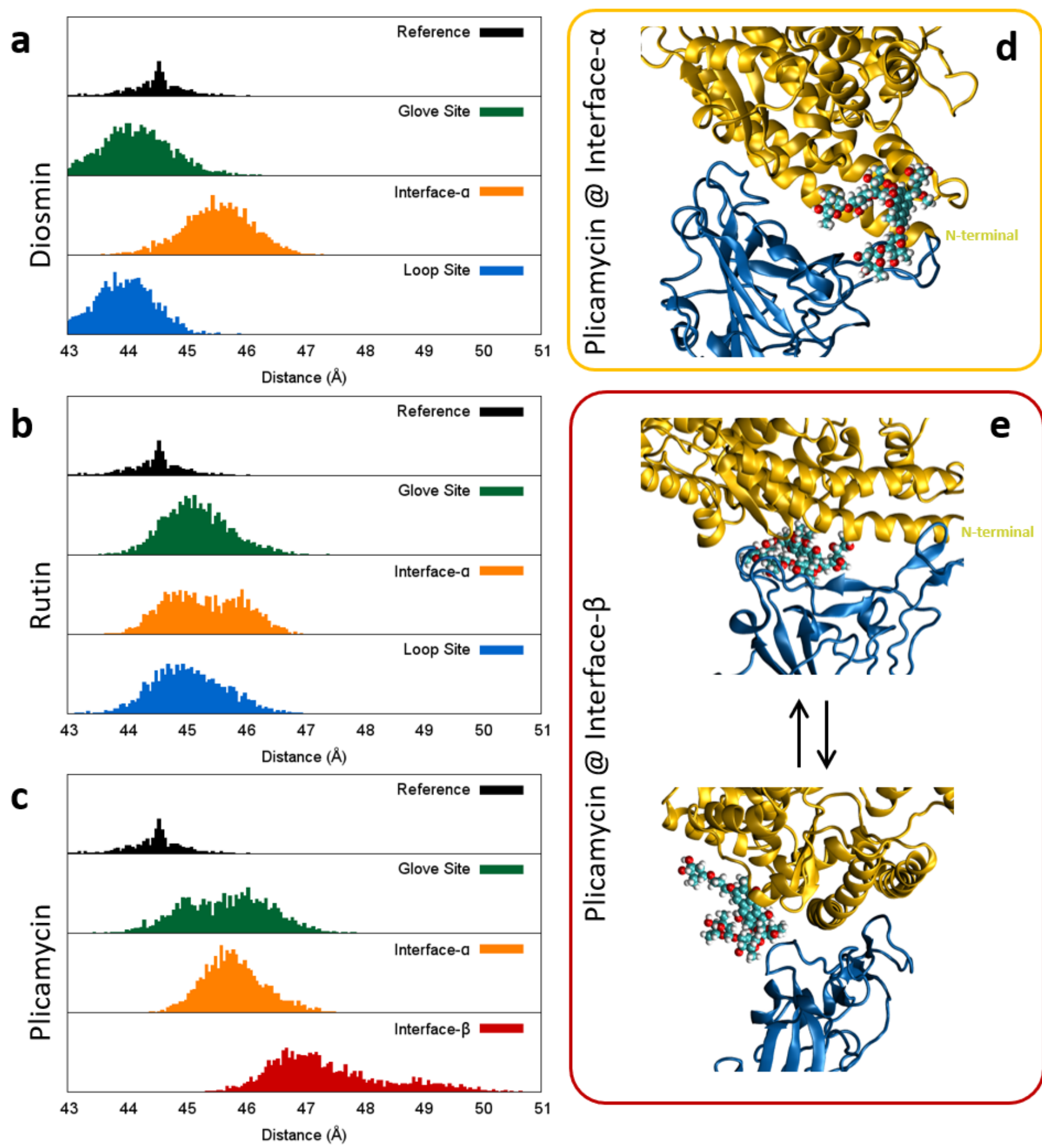

Fig. 3 The distance between RBD and ACE2 is modified by the interacting drugs. Distribution function of the RBD/ACE2-(PD) distance in presence of diosmin (a), rutin (b) and plicamycin (c) at the different binding modes. The results of the RBD/ACE2 in absence of any drug (reference) is also shown for comparison. d, Representative snapshot of plicamycin at the interface- $\alpha$. e, Representative snapshots of the two plicamycin conformations in equilibrium at the interface- $\beta$.

A most useful indicator to quantify the effects of the drug on the RBD/ACE2 complex is the distribution of the distance between their centers of mass at the interface area (see Supplementary Information for the full definition and Supplementary Figure 2 for the corresponding time series), since such distance increases 
when weakening the protein-protein interactions. Representative snapshots extracted from the different MD trajectories are also provided in Figure 3d,e and Supplementary Figure 5, giving a pictorial view of the induced destabilization. In all cases under study it becomes apparent that the distribution of the distance becomes much broader upon the addition of the drug as compared to the reference, hence indicating a noninnocent effect on the RBD/ACE2 interface. However, in the case of diosmin (Figure 3a) both loop and glove sites have no noticeable effect in destabilizing the complex, while the maximum of the distribution is even slightly shifted to shorter distances compared to the reference. As expected, due to the better exposition to the PD area, the interface- $\alpha$ interaction mode, instead, presents a slight increase of about $2 \AA$ of the distribution maximum. However, the global efficiency of diosmin as a valuable ACE2 inhibitor appears quite limited.

Conversely, rutin (Figure 3b) shows clearly improved properties as identified by the fact that all the three interaction modes: loop, glove, and interface- $\alpha$, induce a considerable increase of the distance between the centers of mass, and hence are indicative of the weakening of the protein-protein interactions. Interestingly, the distribution for the interface- $\alpha$ presents a secondary maximum at larger distance, that points to the emergence of a conformational equilibrium and hence an even more evident destabilization. Thus, this fact also confirms the peculiar role played by interface- $\alpha$ binders as opposed to the other sites.

Finally, plicamycin (Figure 3c) definitively appears as the most promising compound. In fact, it presents a novel interaction mode, interface- $\beta$, that is directly facing the RBD interaction area, and that can also be achieved by the sliding of the ligand from the less efficient and spatially close loop site. All the interaction modes are correlated to a noticeable increase of the protein-protein distance while a double maximum structure is also evident for the glove site. As far as the novel intterface- $\beta$ mode is concerned, we observe not only a larger shift of the distribution maximum (more than $3 \AA$ ) but also and especially the emergence of a strongly asymmetry in the distribution with a tail extending noticeably on the longer distance region. Furthermore, a very broad, but still evident, secondary maximum appears at a distance of more than $5 \AA$ A from the reference. The effects of plicamycin on the RBD/ACE2 complex can also be appreciated by the analysis of representative snapshots for interface- $\alpha$ (Figure $3 \mathrm{~d}$ ) that clearly evidences the positioning of the drug at the interface between the two proteins, and for interface- $\beta$ (Figure 3e), in which the presence of an even more open form already visualize a partial disruption of the RBD/ACE2 complex.

In order to better understand those global effects, we also perform a detailed analysis of the specific RBD/ACE2 interactions that are perturbed by the presence of the drugs, in particular the favorable polar interactions that assure the protein-protein binding. The equilibrium MD of the native RBD/ACE2 complex has allowed to confirm the amino acids interacting between the two proteins, as shown in Figure 4. Unsurprisingly, the most important amino acids assuring the interactions are placed at the interface- $\alpha$ and $-\beta$ and are mostly acting through hydrogen bonding, as confirmed by different independent studies. ${ }^{15,22}$ Figures $4 \mathrm{a}-$ c report the difference of the average number of hydrogen bonds per each ACE2 amino acid in presence or in absence of the drug is reported. Globally, these parameters confirm the tendency already evidenced in Figure 3 and indeed, diosmin, especially in glove and loop sites, is producing a less important perturbation compared to the other ligands, while the number of hydrogen bonds weakened by diosmin at the interface$\alpha$, and especially in the N-terminal region of the PD, is clearly more important (see Supplementary Table 1 for more details). The behavior of rutin is similar, however, the weakening of interactions taking place further away from the N-terminal area is more evident (see Supplementary Table 2 for more details). In contrast, once again, a different behavior is observed for plicamycin, especially at interface- $\beta$. In this case, hydrogen bonds encompassing the whole PD region are significantly weakened. In particular, for this specific binding site, one should point out the almost total disruption of the hydrogen bonds Asp355 $\cdots$ Thr500 and Lys353 $\cdots$ Gly502, although in this latter case the strong interaction with Gly502 is replaced by several weak hydrogen bonds with other amino acids (see Supplementary Table 3). In addition, we observe that the drug also weakens indirect hydrogen bonds, i.e. formed through a bridging water molecule, albeit to a lower extent with respect to direct hydrogen bonds (see Supplementary Figure 3). It should be remarked that, independently of the binding site, the drug interacts mainly with ACE2 and not with RBD, through different types of non-covalent interactions as evidenced in Supplementary Figure 4. This confirms our strategy based on blocking solely the domain of ACE2 susceptible of RBD recognition. 

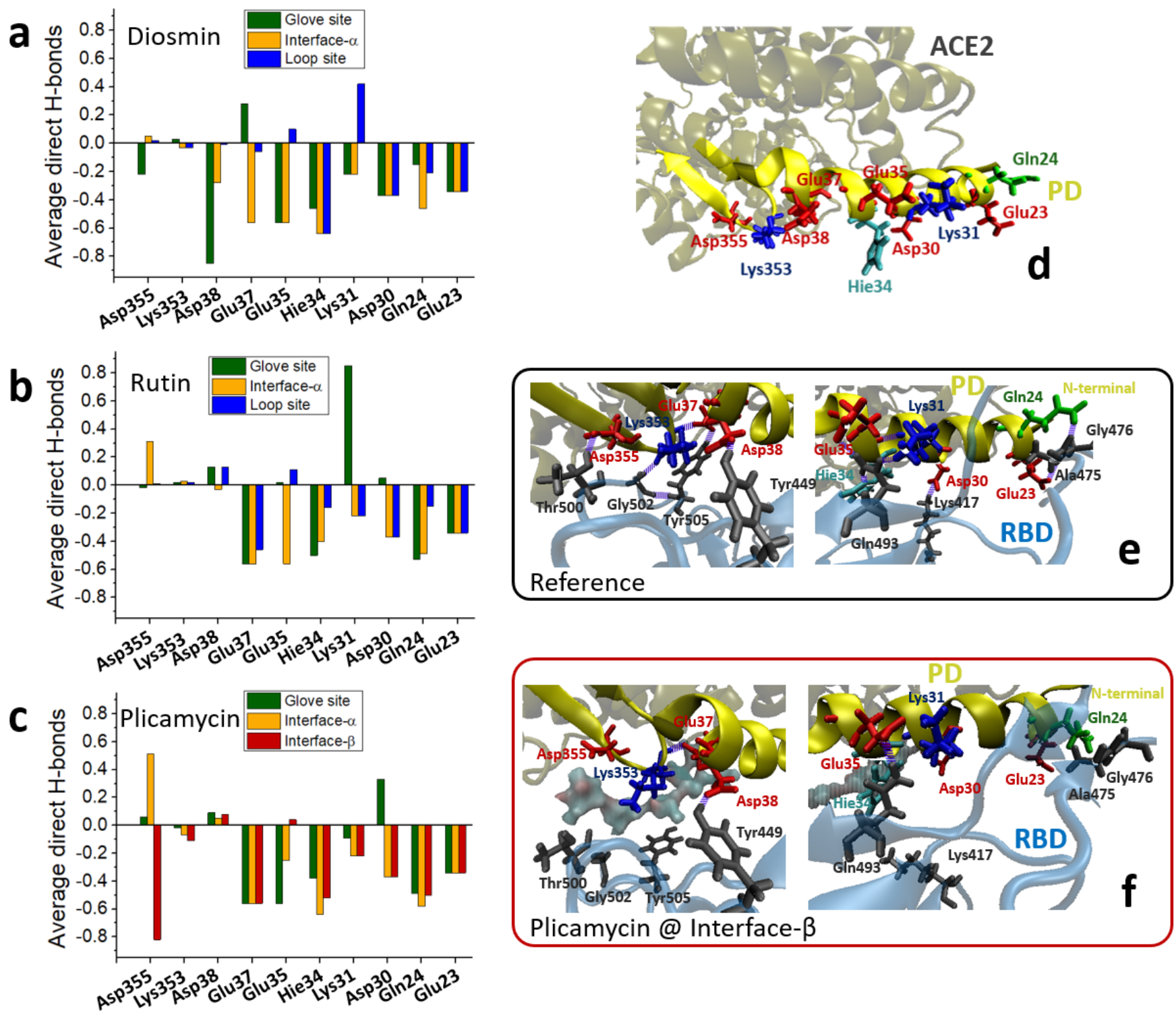

Fig. 4 | The complex RBD/ACE2 hydrogen bonding network is depleted. Histogram showing the increase (positive values) or decrease (negative values) of the direct H-bonds between ACE2-(PD) and RBD for (a) diosmin, (b) rutin and (c) plicamycin, averaged along each trajectory. d, ACE2-(PD) amino acids involved in the formation of direct H-bonds. Color code: red (negatively charged), blue (positively charged), green (polar), cyan (neutral His $\varepsilon$-protonated). e, H-bonding network at the interface- $\beta$ (left) and interface- $\alpha$ (right) sites of the untreated ACE2-(PD)/RBD reference system. RBD amino acid side chains are shown in grey. f, Same as e, now including plicamycin (visualized in surface representation) interacting at the interface- $\beta$.

The fact that plicamycin is effectively acting over all the ACE2/RBD interaction region is essential in explaining the strong destabilization of the protein-protein complex. This can be observed in Figure 4e,f, in which we report the comparison of a representative snapshot showing the hydrogen bond network for the reference complex and plicamycin at the interface- . The breaking of the interactions in both contact regions is evident and is certainly related to the strong destabilization of the complex yielding an open conformation characterized by a much larger protein-protein distance.

The results presented offer a coherent, yet still qualitative, scenario. To better quantify the effect of the best candidate, i.e. plicamycin at interface- we determine the thermodynamic properties of the RBD/ACE2 complex. In order to do so, we calculate the free energy profile along the distance between the center of mass of the two proteins, in presence and absence of plicamycin (Figure 5). The free energy profile for the native 
complex is characterized by a rather deep energy well accounting for a binding free energy of about 6.5 $\mathrm{kcal} / \mathrm{mol}$ at $70 \AA ̊$ distance. As expected, no energetic barrier is evidenced for the formation of the complex, at least considering RBD in its active conformation, confirming the high RBD affinity for ACE2. Note that this result represents, to the best of our knowledge, the first computational estimate of the binding free-energy between ACE2 and the SARS-CoV-2 spike protein. When adding plicamycin we first note, coherently with the equilibrium MD, an increase of the distance between the centers of mass corresponding to the minimum free energy. More importantly, the free energy profile becomes distinctly shallower and the binding energy is reduced to about $4.5 \mathrm{kcal} / \mathrm{mol}$ at $70 \AA$ distance, hence indicating a clear destabilization of the RDB/ACE2 complex. Interestingly, a secondary, less stable minimum at shorter distance is also evidenced, justifying, together with the shallow free energy profile, the two conformations observed by equilibrium MD and the detection of a semi-dissociated conformation.
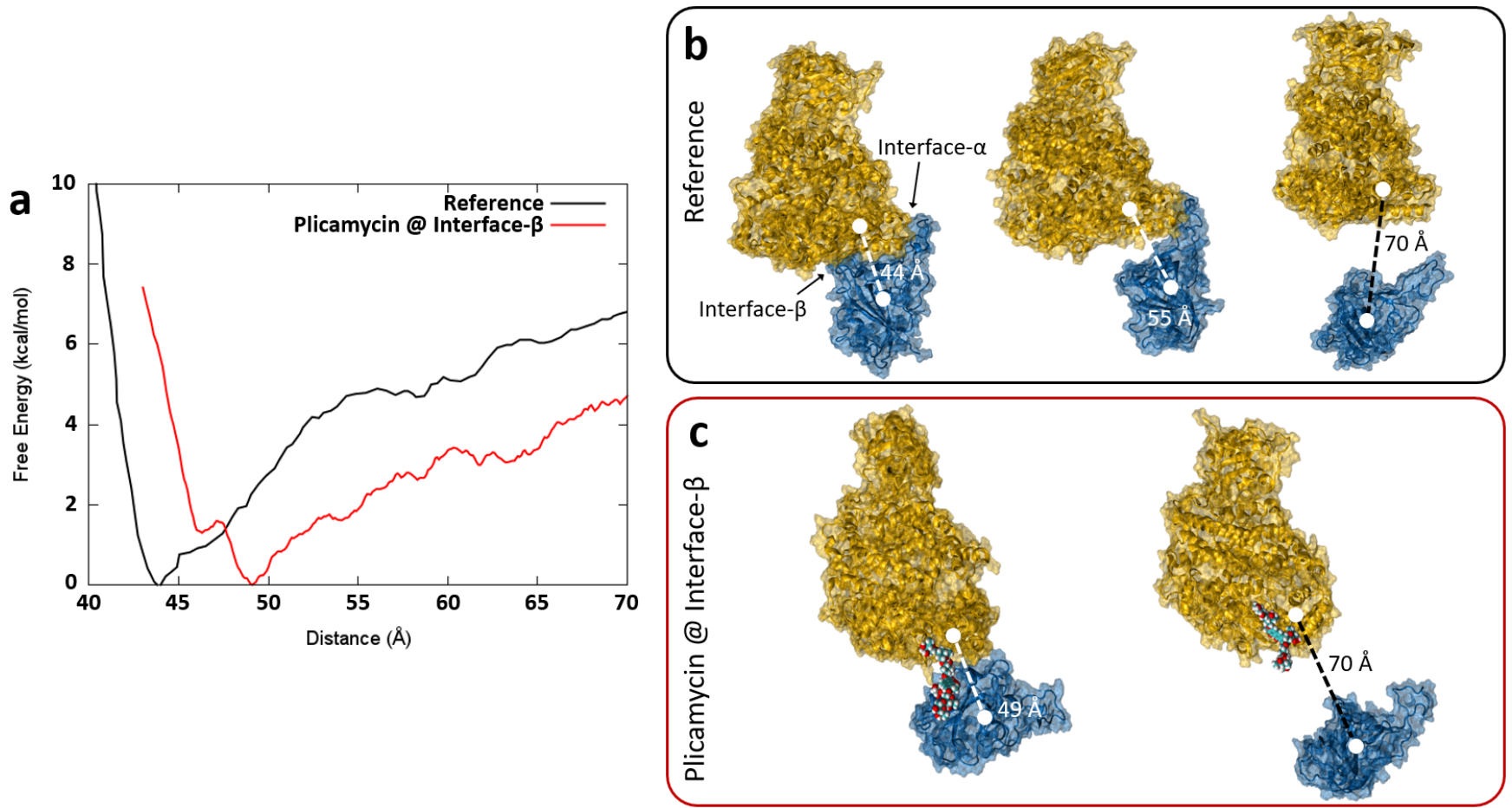

Fig. 5 | Plicamycin reduces considerably the RBD/ACE2 binding free energy. a, Free energy profiles of the RBD/ACE2 complex in absence and in presence of plicamycin at interface- $\beta$. b. Snapshots of the reference system at its free energy minimum, when detaching at interface- $\beta$, and when completely separated. $\mathbf{c}$, Snapshots of the complex in presence of plicamycin at interface- $\beta$, at its free energy minimum and separated.

\section{Discussion}

The very favorable and strong interaction between SARS-CoV-2 spike protein, through its active RBD, and ACE2 represents a peculiarity of this coronavirus that should be correlated to its extremely high transmissibility rate, and hence to its dangerousness, even as compared to the previous SARS-CoV. ${ }^{17}$ This affinity is mostly due to the presence of an extended network of favorable hydrogen bonds, encompassing the rather spread N-terminal PD of ACE2, as coherently confirmed by our results and other independent studies. 15,16,20,30 The estimation of the binding free energy of the RBD/ACE2 complex also points to very strong and favorable interactions.

Since ACE2 constitutes the entry point of SARS-CoV-2 in human cells, its inhibition and the further weakening of the RBD/ACE2 complex formation represents a possible therapeutic strategy to be pursued. However, suitable drugs should possess defined characteristics. Especially, they should form strong and specific interactions with the PD region, and they should not interact with ACE2 catalytic domain to avoid serious secondary effects. As shown by molecular docking, we propose an ensemble of glycosylated drugs, already available, that present different interactions modes with ACE2. MD simulations have clearly shown that while almost 
all the chosen compounds have non-negligible effects in weakening the RBD/ACE2 interaction, as witnessed by the wide distribution of the distance between the centers of mass of the proteins, and by the analysis of the hydrogen bonding network, their efficiency may vary considerably. In particular, the aureolic acid plicamycin clearly stands out as the lead compound. Its efficacy is due to its capacity of perturbing almost all the PD region of ACE2, considerably disrupting the hydrogen bonding network at both interfaces $(-\alpha$ and $-\beta)$. Such an efficiency is already evident at the equilibrium MD by the appearance of partially dissociated conformations presenting a larger protein-protein distance, being the interaction through almost all the PD broken. This qualitative behavior is also confirmed by the binding free energy profile which, when compared with that of the native complex, yields an increased protein-protein distance corresponding to the minimum free energy, while the thermodynamic driving force for the complexation is reduced by ca. $30 \%$ ( $2 \mathrm{kcal} / \mathrm{mol}$ ) at $70 \AA$ distance. Indeed, calculations show that unbinding of the RBD/ACE2 complex would preferably start from interface- $\beta$ detachment, further suggesting the suitability of plicamycin (Figure 5b,c).

From a molecular point of view, the antibiotic plicamycin, also known as mithramycin, appears as a promising agent that could be used to prevent viral infection and hence, potentially, acts as an antiviral agent capable of limiting the virus diffusion in the organism, reducing its virulence and the morbidity of the SARS-CoV-2 pathogen. Plicamycin (also known as mithramycin) being already commercially and clinically approved, ${ }^{31}$ tests to confirm its efficacity should be considered as a top-most priority, to be performed in vitro and in vivo. This should also include the assessment of its side effects such as hepatotoxicity ${ }^{32}$, that in spite to be usually transient and asymptomatic, it could limit its therapeutic use in certain patients with limited hepatic function. Indeed, the potent RBD/ACE2 inhibition clearly demonstrated in the present work, and the vast knowledge of its pharmacology and pharmacokinetics, studied for decades, 33 known to be very active in lungs and esophagus, ${ }^{34}$ makes this drug a very attractive opportunity to treat severe COVID-19 cases. This is especially relevant in the context of emergency and urgency caused by the 2020 COVID-19 pandemic outbreak. In addition, it shall be mentioned that related aureolic acid compounds such as durhamycin $\mathrm{A}^{35}$ and chromomycin ${ }^{36}$ have already shown antiviral activity against HIV.

In addition to specifically pinpoint plicamycin, we also established on a firm basis the interactions between RBD and ACE2, including for the first time the determination of the binding free energy profile, moreover evidencing the most important amino acids that should be targeted to achieve an efficient weakening of the RBD/ACE2 complex formation. Such knowledge boosts the understanding of the SARS-CoV-2 viral infection and can be efficiently used, in a long-term period, for rational molecular design procedures to enhance the efficacy of novel or existing drugs. The clear identification of the molecular bases of RBD/ACE2 complexation and of its inhibition could also help in devising strategies to contrast possible mutations that could lead to resistant viral strains.

\section{Methods}

Our multi-scaling technique including a docking study, followed by MD and PMF simulations, is described hereafter. Details are provided in the SI.

Docking study. To investigate the possible binding modes of the proposed drugs, a docking study considering ACE2 was performed using the Autodock Vina software. ${ }^{37}$ Prior to virtual screening, the 3D geometry of each drug was built with Discovery Studio 2.1 program. The same program was used to add hydrogen atoms and assign bond orders, hybridization and charges to ACE2, extracted from the PDB ID 6M17.22 All drugs rotatable bonds were allowed to rotate freely. For each drug, 50 independent calculations including the lowest 20 binding energies (1000 structures in total) were scrutinized for statistical analysis of the binding pockets and to select representative geometries to run the following molecular dynamics simulations.

All-atom molecular dynamics (MD) simulations. The structure of the RBD/ACE2 complex was extracted from the PDB ID $6 \mathrm{M} 17$, adding the previously selected drug geometry (from docking) and deleting the ACE2 C-terminal $\alpha$-helix to diminish the computational expenses while not hampering the proper description of any ACE2 functional domain. After solvating with water molecules to build a cubic box and adding the corresponding $\mathrm{K}^{+}$counter ions to achieve neutrality, this procedure resulted in the setup of 10 systems, including the RBD/ACE2 reference (without any drug) and three RBD/ACE2/drug starting structures - corresponding to different ACE2/drug binding pockets - for each of the three selected drugs. All the MD simulations reported herein were run using the NAMD ${ }^{38}$ code at $300 \mathrm{~K}$ and $1 \mathrm{~atm}$, with the Amber99SB force field ${ }^{39-41}$ to describe the proteins and TIP3 $\mathrm{P}^{42}$ water molecules. The force field of each drug has been parameterized through the GAFF procedure. ${ }^{43}$ $\mathrm{VMD}^{44}$ was used for visualization, inspection and analysis. 
Potential of mean force (PMF) calculations. The PMF energy profile was calculated by applying a recently developed combination of metadynamics ${ }^{45}$ and adaptative biased force (eABF), ${ }^{46}$ meta-eABF 47,48 implemented in the NAMD code. ${ }^{38}$ It was applied, for comparison purposes, to the RBD/ACE2 reference and to the same system including plicamycin in the interface$\beta$ binding pocket.

\section{References}

1. Watkins, J. Preventing a covid-19 pandemic. The BMJ 368, doi 10.1136/bmj.m810 (2020).

2. Tang, B. et al. Estimation of the Transmission Risk of the 2019-nCoV and Its Implication for Public Health Interventions. J. Clin. Med. (2020). doi: $10.3390 / \mathrm{jcm} 9020462$

3. Cao, Z. et al. Estimating the effective reproduction number of the 2019-nCoV in China. medRxiv 2020.01.27.20018952 (2020). doi: $10.1101 / 2020.01 .27 .20018952$

4. Bedford, J. et al. COVID-19: towards controlling of a pandemic. The Lancet 395, 1015-1018 (2020).

5. Shi, H. et al. Radiological findings from 81 patients with COVID-19 pneumonia in Wuhan, China: a descriptive study. Lancet Infect. Dis. (2020). doi:10.1016/S1473-3099(20)30086-4

6. Lai, C. C., Shih, T. P., Ko, W. C., Tang, H. J. \& Hsueh, P. R. Severe acute respiratory syndrome coronavirus 2 (SARS-CoV-2) and coronavirus disease-2019 (COVID-19): The epidemic and the challenges. International Journal of Antimicrobial Agents (2020). doi:10.1016/j.jjantimicag.2020.105924

7. Kooraki, S., Hosseiny, M., Myers, L. \& Gholamrezanezhad, A. Coronavirus (COVID-19) Outbreak: What the Department of Radiology Should Know. J. Am. Coll. Radiol. (2020). doi:10.1016/j.jacr.2020.02.008

8. Liu, Y., Gayle, A. A., Wilder-Smith, A. \& Rocklöv, J. The reproductive number of COVID-19 is higher compared to SARS coronavirus. Journal of travel medicine (2020). doi:10.1093/jtm/taaa021

9. Yuen, K. S., Ye, Z. W., Fung, S. Y., Chan, C. P. \& Jin, D. Y. SARS-CoV-2 and COVID-19: The most important research questions. Cell Biosci. (2020). doi:10.1186/s13578-020-00404-4

10. Wilder-Smith, A., Chiew, C. J. \& Lee, V. J. Can we contain the COVID-19 outbreak with the same measures as for SARS? The Lancet Infectious Diseases (2020). doi:10.1016/S1473-3099(20)30129-8

11. Dong, E., Du, H. \& Gardner, L. An interactive web-based dashboard to track COVID-19 in real time. The Lancet Infectious Diseases (2020). doi:10.1016/S1473-3099(20)30120-1

12. Andersen, K. G., Rambaut, A., Lipkin, W. I., Holmes, E. C. \& Garry, R. F. The proximal origin of SARS-CoV-2. Nat. Med. (2020). doi:10.1038/s41591-020-0820-9

13. Tang, X. et al. On the origin and continuing evolution of SARS-CoV-2. Natl. Sci. Rev. (2020). doi:10.1093/nsr/nwaa036

14. Wang, Q. et al. Structural and functional basis of SARS-CoV-2 entry by using human ACE2. Cell (2020). doi:10.1016/j.cell.2020.03.045

15. Shang, J. et al. Structural basis of receptor recognition by SARS-CoV-2. Nature (2020). doi:10.1038/s41586-020-2179-y

16. Lan, J. et al. Structure of the SARS-CoV-2 spike receptor-binding domain bound to the ACE2 receptor. Nature (2020). doi:10.1038/s41586-020$2180-5$

17. Wrapp, D. et al. Cryo-EM structure of the 2019-nCoV spike in the prefusion conformation. Science (80-. ). (2020). doi:10.1126/science.aax0902

18. Chen, H. \& Du, Q. Potential natural compounds for preventing SARS-CoV-2 (2019-nCoV) infection. Preprints (2020). doi:10.20944/preprints202001.0358.v3

19. Hognon, C. et al. Role of RNA Guanine Quadruplexes in Favoring the Dimerization of SARS Unique Domain in Coronaviruses. bioRxiv 2020.04.07.029447 (2020). doi:10.1101/2020.04.07.029447

20. Spinello, A., Saltalamacchia, A. \& Magistrato, A. Is the Rigidity of SARS-CoV-2 Spike Receptor-Binding Motif the Hallmark for Its Enhanced Infectivity? An Answer from All-Atoms Simulations. (2020). doi:10.26434/CHEMRXIV.12091260.V2

21. Lai, Z. W., Hanchapola, I., Steer, D. L. \& Smith, A. I. Angiotensin-converting enzyme 2 ectodomain shedding cleavage-site identification: Determinants and constraints. Biochemistry (2011). doi:10.1021/bi200525y

22. Yan, R. et al. Structural basis for the recognition of the SARS-CoV-2 by full-length human ACE2. Science (80-.). 367, doi: 10.1126/science.abb2762 (2020).

23. Oudit, G. Y., Crackower, M. A., Backx, P. H. \& Penninger, J. M. The role of ACE2 in cardiovascular physiology. Trends in Cardiovascular Medicine (2003). doi:10.1016/S1050-1738(02)00233-5 
24. Crackower, M. A., Sarao, R., Oliveira-dos-Santos, A. J., Da Costa, J. \& Zhang, L. Angiotensin-converting enzyme 2 is an essential regulator of heart function. Nature (2002). doi:10.1038/nature00786

25. Böhmer, C. et al. The serum and glucocorticoid inducible kinases SGK1-3 stimulate the neutral amino acid transporter SLC6A19. Cell. Physiol. Biochem. (2010). doi:10.1159/000315092

26. Verity, R. et al. Estimates of the severity of coronavirus disease 2019 : a model-based analysis. Lancet Infect. Dis. 3099, 1-9 (2020).

27. Vincent, J.-L. \& Taccone, F. S. Understanding pathways to death in patients with COVID-19. Lancet Respir. Med. doi: 10.1016/S22132600(20)30165-X (2020). doi:10.1016/S2213-2600(20)30165-X

28. Yuan, J., Li, M., Lv, G. \& Lu, Z. K. Monitoring Transmissibility and Mortality of COVID-19 in Europe. Int. J. Infect. Dis. doi: 10.1016/j.ijid.2020.03.050 (2020). doi:10.1016/j.ijid.2020.03.050

29. Prabakaran, P., Xiao, X. \& Dimitrov, D. S. A model of the ACE2 structure and function as a SARS-CoV receptor. Biochem. Biophys. Res. Commun. (2004). doi:10.1016/j.bbrc.2003.12.081

30. Lupala, C. S. et al. Computational simulations reveal the binding dynamics between human ACE2 and the receptor binding domain of SARS-CoV2 spike protein. bioRxiv (2020). doi:10.1101/2020.03.24.005561

31. Taylor, D. J., Parsons, C. E., Han, H., Jayaraman, A. \& Rege, K. Parallel screening of FDA-approved antineoplastic drugs for identifying sensitizers of TRAIL-induced apoptosis in cancer cells. BMC Cancer (2011). doi:10.1186/1471-2407-11-470

32. Sissung, T. M. et al. Severe hepatotoxicity of mithramycin therapy caused by altered expression of hepatocellular bile transporters. Mol. Pharmacol. 96, 158-167 (2019).

33. Lombó, F., Menéndez, N., Salas, J. A. \& Méndez, C. The aureolic acid family of antitumor compounds: structure, mode of action, biosynthesis, and novel derivatives. Appl. Microbiol. Biotechnol. 73, 1-14 (2006).

34. Zhang, M. et al. Mithramycin represses basal and cigarette smoke-induced expression of ABCG2 and inhibits stem cell signaling in lung and esophageal cancer cells. Cancer Res. 72, 4178-4192 (2012).

35. Jayasuriya, H. et al. Durhamycin A, a potent inhibitor of HIV Tat transactivation. J. Nat. Prod. 65, 1091-1095 (2002).

36. Bianchi, N. et al. Targeting of the HIV-1 long terminal repeat with chromomycin potentiates the inhibitory effects of a triplex-forming oligonucleotide on Sp1-DNA interactions and in vitro transcription. Biochem. J. 326, 919-927 (1997).

37. Trott, O. \& Olson, A. J. AutoDock Vina: Improving the speed and accuracy of docking with a new scoring function, efficient optimization, and multithreading. J. Comput. Chem. (2009). doi:10.1002/jcc.21334

38. Phillips, J. C. et al. Scalable molecular dynamics with NAMD. J. Comput. Chem. 26, 1781-1802 (2005).

39. Maier, J. A. et al. ff14SB: Improving the Accuracy of Protein Side Chain and Backbone Parameters from ff99SB. J. Chem. Theory Comput. 11, 3696-3713 (2015).

40. Galindo-Murillo, R. et al. Assessing the Current State of Amber Force Field Modifications for DNA. J. Chem. Theory Comput. 12, 4114-4127 (2016).

41. Zgarbová, M. et al. Refinement of the Sugar-Phosphate Backbone Torsion Beta for AMBER Force Fields Improves the Description of Z- and BDNA. J. Chem. Theory Comput. 11, 5723-5736 (2015).

42. Jorgensen, W. L., Chandrasekhar, J., Madura, J. D., Impey, R. W. \& Klein, M. L. Comparison of simple potential functions for simulating liquid water. J. Chem. Phys. 79, 926-935 (1983).

43. Wang, J. M., Wolf, R. M., Caldwell, J. W., Kollman, P. A. \& Case, D. A. Development and testing of a general amber force field. J. Comput. Chem. 25, 1157-1174 (2004).

44. Humphrey, W., Dalke, A. \& Schulten, K. VMD: Visual molecular dynamics. J. Mol. Graph. (1996). doi:10.1016/0263-7855(96)00018-5

45. Barducci, A., Bonomi, M. \& Parrinello, M. Metadynamics. Wiley Interdisciplinary Reviews: Computational Molecular Science 1, 826-843 (2011).

46. Zhao, T. et al. The Extended Generalized Adaptive Biasing Force Algorithm for Multidimensional Free-Energy Calculations. J. Chem. Theory Comput. 13, 1566-1576 (2017).

47. Fu, H. et al. Zooming across the Free-Energy Landscape: Shaving Barriers, and Flooding Valleys. J. Phys. Chem. Lett. 9, $4738-4745$ (2018).

48. Fu, H., Shao, X., Cai, W. \& Chipot, C. Taming Rugged Free Energy Landscapes Using an Average Force. Acc. Chem. Res. 52, 3254-3264 (2019). 


\section{Acknowledgments}

Support from the Université de Lorraine and French CNRS is gratefully acknowledged. A.F.-M. is grateful to Generalitat Valenciana and the European Social Fund for a postdoctoral contract (APOSTD/2019/149) and the Ministerio de Ciencia e Innovación (project (CTQ2017-87054-C2-2-P) for financial support. French CNRS and IDRIS national computing center are acknowledged for graciously providing access to computational resources in the framework of the special Covid-19 mobilization under the project "Seek\&Destroy". Part of the calculations have been performed on the LPCT local computing cluster, on the regional ExpLor center in the frame of the project "Dancing Under the Light", and on the local computing cluster of the "Reactivity and Molecular Structure Group" at the Universidad de Alcalá.

\section{Author contributions}

C.G.-I., I.I. and M.M. conceived the project. I.I. and C.G.-I. performed the docking study. C.G.-I. and A.F.-M. did the setup and run the molecular dynamics, that were analyzed by C.G.-I., A.F.-M., C.H., A.M. and M.M. C.H. performed and analyzed the potential of mean force calculations, with the help of T.M.. A.M. and M.M. wrote the manuscript. C.G.-I., A.M. and M.M. made the figures. All authors reviewed and discussed the data and manuscript.

\section{Supplementary information}

Additional results and discussion, extended materials and methods, Supplementary Figs. 1-6, Tables 1-3 and references.

Competing Interests. The authors declare no competing interests.

\section{Materials \& Correspondence}

*Correspondence and requests for materials should be addressed to M.M. (email: marco.marazzi@uah.es) or to A.M. (email: antonio.monari@univ-lorraine.fr). 